\title{
BIBFRAME: MODELO DE DADOS INTERLIGADOS PARA BIBLIOTECAS
}

\section{BIBFRAME: MODELO DE DATOS ENLAZADOS PARA BIBLIOTECAS}

\author{
Rogério Aparecido Sá Ramalho*
}

\begin{abstract}
RESUMO
Introdução: O desenvolvimento tecnológico da última década tem proporcionado novos instrumentos de modelagem, representação e organização de recursos informacionais nas mais diversas áreas do conhecimento. No campo da Ciência da Informação destaca-se o Bibliographic Framework (BIBFRAME) como um novo modelo de dados que possibilita a descrição formal dos relacionamentos entre os recursos. Este modelo está relacionado com as tendências de pesquisa envolvendo Linked Data; Data Science; Publicação Ampliada e Web Semântica.

Objetivo: Fornecer subsídios teóricos que contribuam para uma melhor compreensão do modelo de dados BIBFRAME, descrever seus elementos e apresentar uma discussão sobre os principais desafios e perspectivas de uso deste novo modelo de dados em bibliotecas.

Metodologia: Pesquisa exploratória e descritiva, com abordagem teórica.

Resultados: Foram apresentadas as principais tecnologias, elementos conceituais e características do modelo BIBFRAME e relatados os desafios e perspectivas de uso deste novo modelo de dados em bibliotecas.

Conclusões: O modelo BIBFRAME contribui para uma mudança de paradigma em relação à forma como encaramos os processos de controle, troca e compartilhamento de recursos informacionais, fornecendo um impulso para o reposicionamento das bibliotecas no cenário contemporâneo, a fim de fortalecer atividades relacionadas à identificação e formalização dos relacionamentos entre recursos disponíveis na Web.
\end{abstract}

Palavras-chave: Sistemas de Organización del Conocimiento. Tecnologías de Información y Comunicación. Modelado de datos. Ontologías. Web Semántica.

\section{INTRODUÇÃO:}

Tradicionalmente as bibliotecas sempre ocuparam um papel de destaque como espécie de "cérebro" universal e "guardiões" do saber coletivo da humanidade, onde podemos reaver o que esquecemos e o que ainda não *Doutor em Ciência da Informação pela Universidade Estadual Paulista (Unesp-Marília). Professor do Programa de Pós-Graduação em Ciência da Informação da Universidade Federal de São Carlos (UFSCar). E mail: ramalho@ufscar.br 
sabemos (ECO, 2003). Com o avanço das tecnologias digitais novas possibilidades de representação e organização de informações têm sido propostas no intuito de favorecer um melhor atendimento das necessidades informacionais de uma sociedade, cada vez mais, baseada em interações virtuais.

Inegavelmente vivemos um momento de mudança e as bibliotecas, assim como a maioria das instituições sociais, precisam ser repensadas sob novas perspectivas, favorecendo a incorporação de novas tecnologias aos seus processos e métodos de trabalho. Conforme destaca Hallo et al. (2016) atualmente torna-se imprescindível que as bibliotecas disponibilizem conteúdos na Web e utilizem novos artefatos tecnológicos como ontologias, descrições semânticas de conteúdos, links de dados e novas formas de colaboração baseadas no uso de redes sociais, comunidades especializadas, wikis, jogos colaborativos e mashups.

Ao longo da última década novos instrumentos de modelagem, representação e organização de recursos informacionais têm sido propostos nas mais variadas áreas do conhecimento. No campo da Ciência da Informação destaca-se o Bibliographic Framework (BIBFRAME) um novo modelo de dados que favorece a descrição formal dos relacionamentos existentes entre os recursos por meio de links, indo ao encontro das novas tendências de pesquisas como: Linked Data; Data Science; Publicação Ampliada e Web Semântica;

Apesar das potencialidades oferecidas pelo BIBIFRAME identifica-se uma carência de pesquisas, principalmente no âmbito nacional, focadas no estudo deste modelo. Este artigo tem como objetivo apresentar subsídios teóricos que contribuam para uma melhor compreensão do modelo de dados BIBFRAME, descrever seus elementos e apresentar uma discussão sobre os principais desafios e perspectivas de uso deste novo modelo de dados em bibliotecas.

A metodologia utilizada caracteriza-se como uma pesquisa exploratória e descritiva, com abordagem teórica realizada a partir da análise dos documentos disponibilizados pela Library of Congress (EUA), e demais colaboradores, que tem direcionado o desenvolvimento do modelo BIBFRAME. 
Justifica-se a pesquisa devido à necessidade de um melhor entendimento deste novo modelo de dados e a escassez de materiais pertinentes a temática. Assim, espera-se contribuir para uma maior discussão sobre os desafios e perspectivas relacionados a disponibilização e interligação de dados em bibliotecas.

\title{
1 BREVE RESGATE HISTÓRICO DO USO DE TECNOLOGIAS DE REPRESENTAÇÃO EM BIBLIOTECAS
}

As bibliotecas têm uma longa e rica história de uso de tecnologias de representação e organização. Na década de 1960 a Library of Congress - LC (EUA) liderou esforços para o desenvolvimento do formato Machine-Readable Cataloging (MARC), o qual se tornou o primeiro padrão de intercâmbio de dados para a catalogação automatizada. O MARC foi criado para o compartilhamento de informações catalográficas em formato eletrônico e após meio século desde a sua concepção inicial manteve-se praticamente inalterado, beneficiando a comunidade de biblioteconomia com um período substancial de estabilidade do modelo de dados (LIBRARY OF CONGRESS, 2012).

\begin{abstract}
Ao longo das últimas décadas a LC tem promovido adequação do formato à tipologia documental, caso do USMARC em 1983. Posteriormente, na seqüência evolutiva, em busca de uma maior integração entre variantes do formato, e também de cobertura na codificação documental, soma esforços cooperativos entre Network Development and MARC Standards Office, Library of Congress, com Standards, Intellectual Management Office, Library and Archives Canada e a National Bibliographic Service, British Library, que harmonizam seus formatos em um único denominado MARC21 (MODESTO, 2007, online).
\end{abstract}

A partir da criação e popularização da linguagem EXtensible Markup Language (XML), observou-se uma nova possibilidade de favorecer 0 intercâmbio de dados entre diferentes formatos. Assim, a partir de uma iniciativa conjunta envolvendo a Library of Congress e o MARC Standards Office foi disponibilizado em 2002 MARC21 XML Schema, mais conhecido como MARCXML.

Inf. Inf., Londrina, v. 21, n. 2, p. 292 - 306, maio/ago., 2016. 
O MARCXML possui exatamente a mesma estrutura do formato MARC21, diferenciando-se apenas na forma de representação a partir do aproveitamento das potencialidades oferecidas pela linguagem XML.

A linguagem XML tem como principal objetivo descrever formalmente os elementos que compõem a estrutura interna de um recurso. Contudo, para formalizar as relações existentes entre diferentes recursos é recomenda a utilização do Resource Description Framework (RDF) que tem como foco principal a representação dos relacionamentos.

Ao longo da última década a linguagem XML passou a ser conhecida no campo da Ciência da Informação, sendo cada vez mais utilizada para a representação de recursos informacionais. Contudo, a tecnologia RDF ainda é pouco explorada. Tal fato pode ser justificado por se tratar de uma tecnologia mais recente e que possui ainda um número restrito de aplicações práticas conhecidas na área.

Entre os novos modelos de representação baseados em RDF pode-se destacar o Metadata Authority Description Schema (MADS) utilizado para a representação de registros de autoridade e o Simple Knowledge Organization System (SKOS) que possibilita a representação da estrutura básica e conteúdo de Sistemas de Organização do Conhecimento. Os elementos do modelo SKOS são declarados a partir de diretivas RDF e podem ser categorizados como: Conceitos, Propriedades e Relações (RAMALHO, 2015).

Em um mundo cada vez mais conectado, onde proliferam-se iniciativas baseadas em dados interligados, torna-se imperativo o desenvolvimento de novos modelos de dados que favoreçam a disponibilização e interligação dos recursos disponíveis em bibliotecas com o ambiente Web. Nesta perspectiva, tem sido desenvolvido o Bibliographic Framework.

\section{BIBLIOGRAPHIC FRAMEWORK INITIATIVE}

O Bibliographic Framework (BIBFRAME) foi lançado oficialmente em maio de 2011 a partir de uma iniciativa da Library of Congress (EUA) com o objetivo de revistar e, a longo prazo, implementar um novo modelo e dados 
para a descrição de recursos bibliográficos que possibilitasse a incorporação das bibliotecas em um novo cenário de dados interligados.

A concepção do modelo BIBFRAME está baseada nas tendências e práticas contemporâneas que incentivam a disponibilização e interligação de dados na Web em formatos que sejam legíveis por máquinas. A medida que a Web evolui, de uma rede de documentos para uma rede de dados interligados, torna-se cada vez mais evidente a necessidade de identificação das entidades envolvidas na criação dos recursos (autores, editores) e dos conceitos (assuntos) relacionados, tais dados são cruciais para que os usuários possam localizar informações na Web (LIBRARY OF CONGRESS, 2012).

Uma entidade, pode ser definida como "algo com caráter unitário e independente; algo com existência independente ou separada; uma abstração, conceito ideal, objeto de pensamento ou objeto transcendental" (INTERNATIONAL FEDERATION OF LIBRARY ASSOCIATIONS, 2009, p. 11, tradução livre),

O modelo BIBFRAME está baseado na representação formal de entidades por meio de Classes RDF, seus elementos precisam ser formalmente identificados a partir da utilização de Uniform Resource Identifier (URI). Conforme apresentado pela Library of Congress (2012, p.3, tradução livre), tais características visam contemplar os principais objetivos que direcionaram a criação do padrão:

1. Diferenciar claramente o conteúdo conceitual e suas manifestações físicas (por exemplo, obras e instâncias);

2. Focar na identificação de forma inequívoca das entidades de informação (por exemplo, as autoridades);

3. Alavancar e expor as relações entre entidades.

O vocabulário BIBIFRAME é formado por um conjunto de Classes e Propriedades que representam formalmente entidades, suas características e relacionamentos. Em abril de 2016 foi publicada uma atualização do vocabulário, denominada como BIBIFRAME 2.0. Considerando as melhorias e avanços identificados em relação a versão anterior, o presente artigo tem como foco de análise esta última versão publicada do modelo, denominando-o 
simplesmente como BIBFRAME. A figura 01 apresenta as principais Classes deste modelo.

Figura 01 - Modelo BIBFRAME 2.0

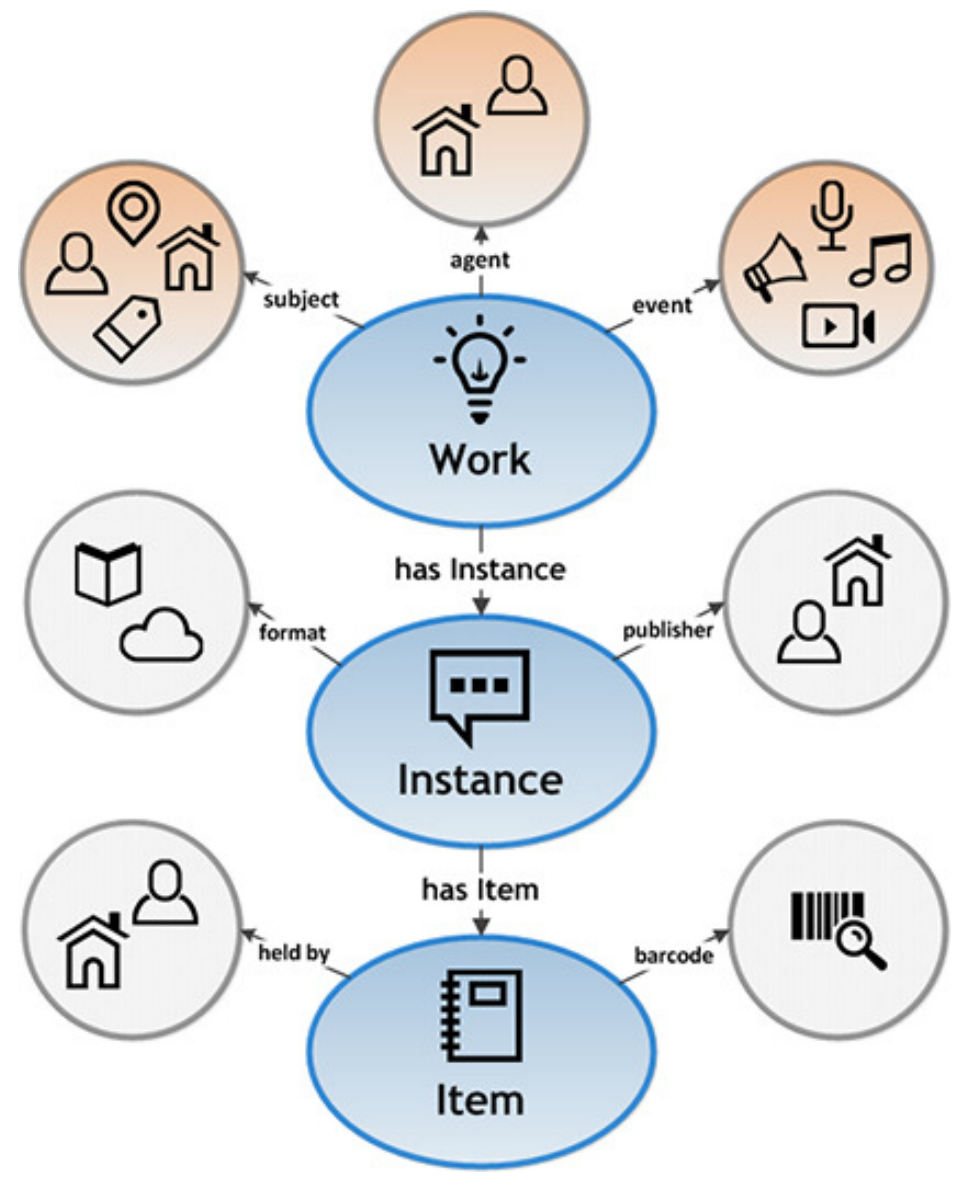

Fonte: Library of Congress (2016, on line).

As três Classes principais do modelo BIBFRAME podem ser descritas sucintamente como: Work (Obra) representa o nível mais alto de abstração e reflete a essência conceitual do recurso catalogado; Instance (Instância) caracteriza uma manifestação e expressão de uma Obra; Item (Item) corporifica uma instância, de forma física ou virtual. Conforme destaca Mitchell (2013) as Classes Work e Item do BIBFRAME são análogas às entidades de mesmo nome previstas nos Functional Requirements for Bibliographic Records (FRBR). Contudo, a Classe Instance pode ser considerada como uma fusão das entidades Expression e Manifestation previstas no FRBR, conforme apresentado na figura 02. 
Figura 02 - Entidades Principais do BIBFRAME e do Grupo 1 do FRBR

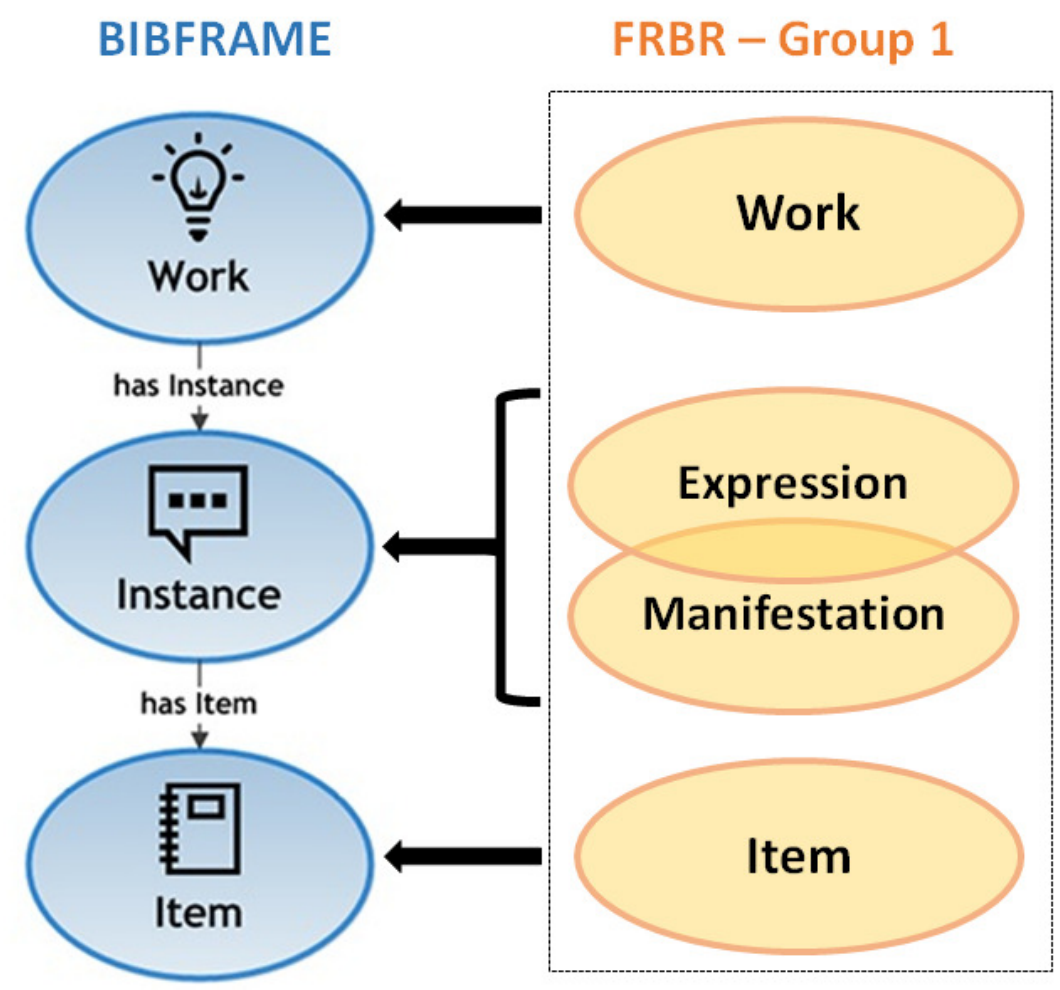

Fonte: Adaptado de Library of Congress (2016, on line).

Esta particularidade da Classe Instance não deve ser interpretada equivocadamente como um afastamento do BIBFRAME em relação ao modelo FRBR que se baseia no Resource Description and Access (RDA), pois no BIBFRAME as relações não são hierárquicas, e sim representadas por meio de grafos RDF, o que favorece uma maior liberdade e simplificação das representações.

Além das três Classes principais (Obra, Instância e Item) é importante destacar que o BIBFRAME também utiliza outras Classes e Subclasses para categorizar as diferentes tipologias de recursos, ultrapassando os limites tradicionais da representação de registros bibliográficos. Conforme descrito pela International Federation of Library Associations and Associations (2005) registro bibliográfico é um conjunto de dados, compostos por entidades, que descrevem ou identificam uma ou mais obras, expressões, manifestações ou itens. 
O BIBFRAME está baseado na formalização dos relacionamentos existentes entre os recursos e não em registros isolados, deste modo possibilita explorar as relações de forma independente, evitando duplicidades de informações e favorecendo a interligação de recursos de naturezas distintas. Baseando-se em um conceito amplo de recurso, além das entidades principais, o BIBFRAME possibilita representar 'Registros de Autoridade', 'Conceitos', 'Lugares' e praticamente qualquer entidade do mundo real que possa ser associada a um identificador controlado e possua um conjunto específico de atributos.

Propriedades são utilizadas para definir formalmente os atributos e relacionamentos e representam os metadados dos recursos. Entre as dezenas de Propriedades previstas no BIBFRAME destacam-se algumas 'Propriedades Gerais'que são herdadas pela maioria das Classes:

- bf:label: propriedade para definição de etiquetas a partir de uma sequência textual;

- bf:identifier: propriedade que especifica um identificador para informações sobre um recurso;

- bf:authorizedAccessPoint: propriedade formulada de acordo com as regras de descrição.

Além destas, as Propriedades bf:hasAnnotation e bf:hasAuthority também podem ser consideradas como 'Propriedades Gerais' devido ao fato que a maioria dos recursos estarem associados anotações (bf:hasAnnotation) e recursos de autoridade (bf:hasAuthority).

O controle de autoridade busca a consistência dos pontos de acesso e correta identificação das entidades responsáveis pelas obras e historicamente é um dos aspectos mais dispendiosos na descrição bibliográfica (BURGER, 1985; GORMAN, 2003; ASSUMPÇÃO; SANTOS, 2012).

Um registro de autoridade pode estar associado a milhares de outros registros, exigindo a atualização de todos caso alguma informação de autoridade seja alterada, gerando retrabalho e um alto custo de manutenção.

O BIBIFRAME baseia-se no uso de links e identificadores controlados, favorecendo a diminuição dos custos associados a manutenção de recursos de 
autoridade, pois evita o retrabalho e a duplicidade de informações. Além disso, o uso de links possibilita uma maior visibilidade dos recursos na Web, extrapolando os limites tradicionais dos catálogos das bibliotecas.

A Figura 03 apresenta graficamente um exemplo simplificado de duas instâncias e um registro de autoridade relacionado a Obra 'FRBR: Final Report'. Em destaque é apresentado o identificador associado ao 'IFLA-StudyGroup', que se encontra disponível no link $<$ http://id.loc.gov/authorities/names/nr98013265>.

Figura 03 - Entidades Principais do BIBIFRAME e do Grupo 1 do FRBR

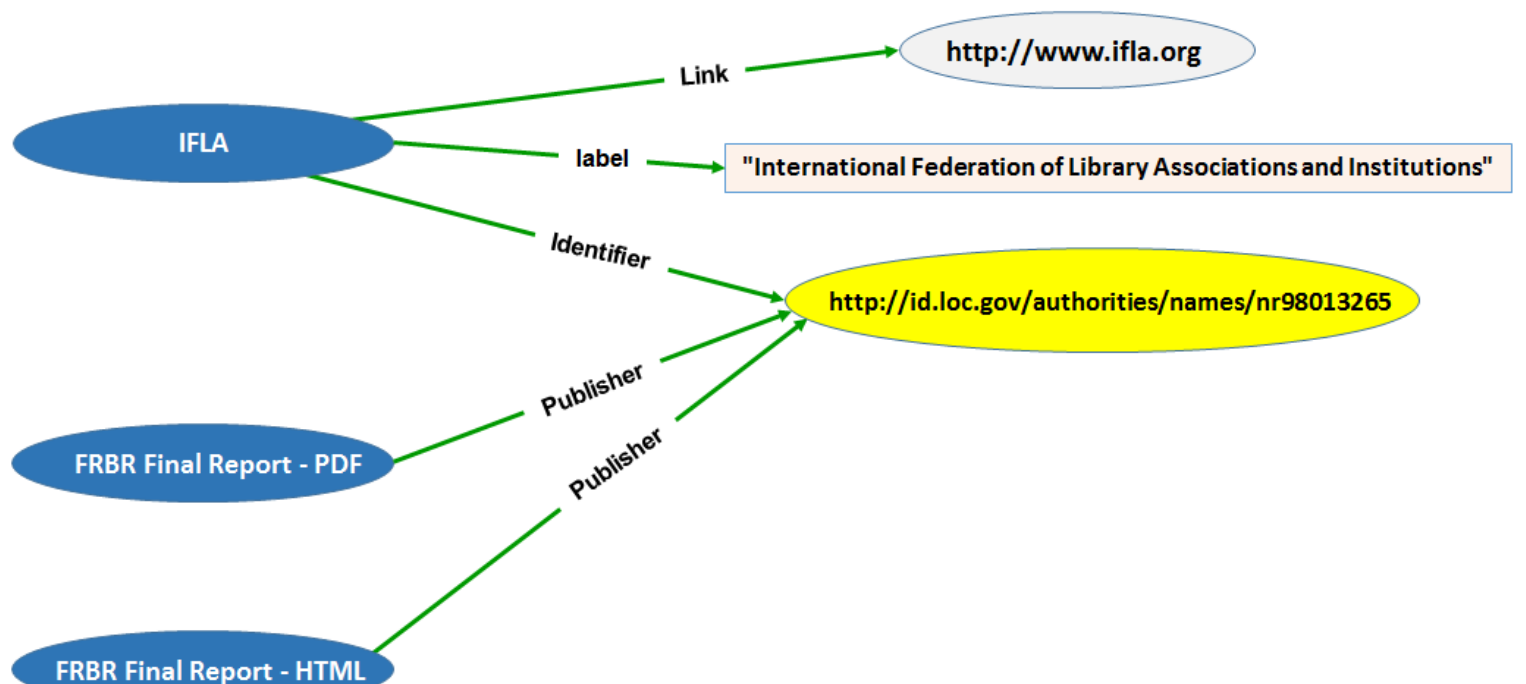

FRBR Final Report - HTML

Fonte: Elaborado pelo autor.

A Figura 03 apresenta três Instâncias, duas correspondentes a diferentes tipos de manifestações da uma Obra ('FRBR Final Report - PDF'; 'FRBR Final Report - HTML') e uma terceira que correspondente ao recurso de autoridade 'IFLA'. Com o objetivo de facilitar a visualização foram ignoradas as outras propriedades das Instâncias, apresentando apenas as Propriedades que relacionam com o recurso de autoridade responsável pela publicação ('Publisher') e três propriedades que descrevem o recurso de autoridade (link; label; Identifier). Como pode ser observado as duas Instâncias 
correspondentes a tipos de manifestações estão vinculadas ao identificador de um mesmo recurso de autoridade, caso alguma Propriedade deste recurso seja alterada automaticamente serão atualizados todos os recursos que vinculados ao seu identificador.

Outra vantagem da utilização de URIs para identificar e disponibilizar os relacionamentos existentes entre os recursos é a possibilidade de interligar dados armazenados em diferentes fontes, contribuindo para a construção de padrões e vocabulários distribuídos, e favorecendo uma maior visibilidade dos recursos disponíveis nos catálogos das bibliotecas.

Conforme destaca a Library of Congress (2012) os registros de autoridade representados em BIBFRAME não são projetados para competir ou substituir as iniciativas de controle de autoridade existentes, mas sim fornecer uma camada de abstração padronizada acima dos diferentes esforços de controle de autoridade baseados na Web para torna-los ainda mais eficazes. Assim, os recursos de autoridade assumem um papel de destaque no modelo BIBFRAME, conforme apresentado na figura 04.

Figura 04 - Representação gráfica de elementos do Modelo BIBFRAME

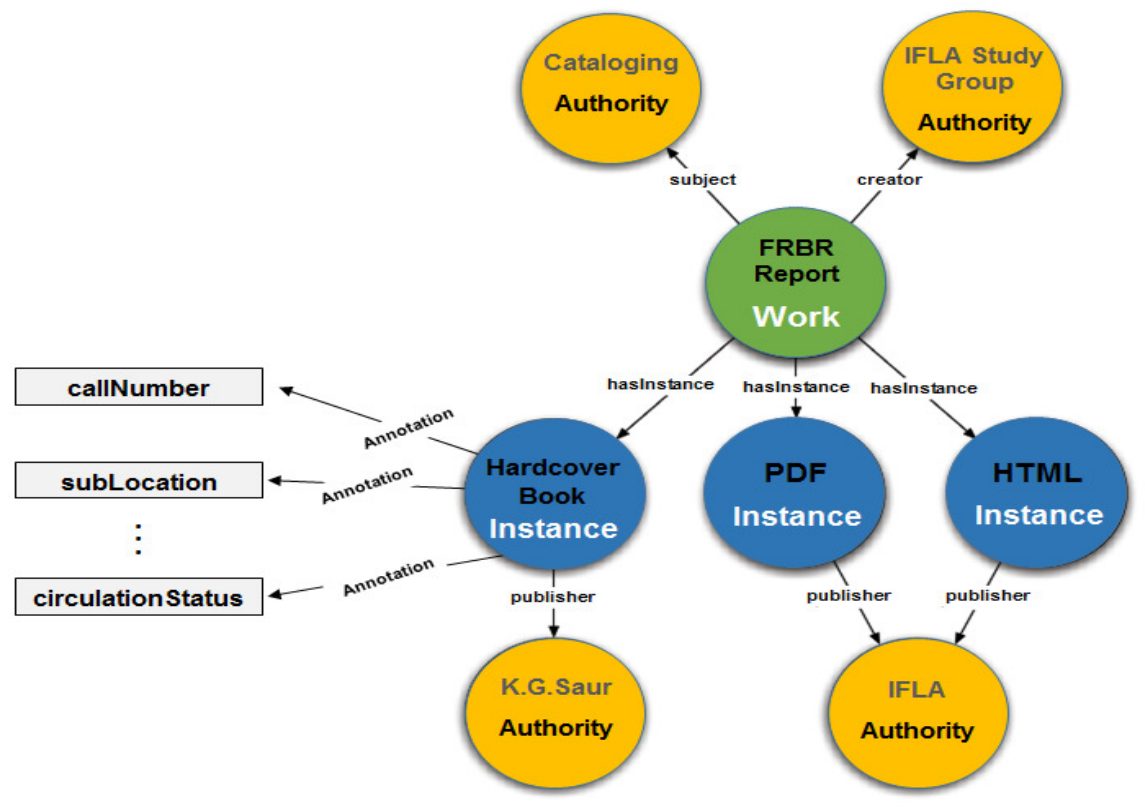

Fonte: Elaborado pelo autor 
A figura 04 apresenta a representação gráfica de um Obra (Work) que é identificada como 'FRBR Report' e possui três Instâncias ('HardCover Book'; (PDF'; HTML'). A Obra está associada a dois recursos de autoridade ('Cataloging'; 'IFLA Study Group), enquanto as Instâncias estão associadas a outros dois recursos de autoridade ('K. G. Saur'; 'IFLA'). Na figura pode-se observar que a autoridade responsável pela publicação da Instância 'HardCover Book' é diferente da autoridade responsável pela publicação das outras duas Instâncias da mesma Obra. Também pode ser observado que a Instância 'HardCover Book' está vinculada a três Propriedades ('callNumber', 'subLocation', 'circulationStatus), a partir da atribuição dos valores correspondentes às Propriedades de uma Instância temos a corporificação de um Item.

Conforme pode ser observado o BIBFRAME deve ser capaz de acomodar um número abrangente de modelos de representação e padrões de metadados, favorecendo o compartilhamento e interligação dos relacionamentos no ambiente Web sem limitar as atividades de catalogação. Conforme destaca a Library of Congress (2012) embora seja importante compreender o BIBIFRAME a partir de visão mais abrangente que ultrapassa os limites tradicionais das bibliotecas, também é imprescindível que os profissionais envolvidos nas bibliotecas se identifiquem com a proposta deste novo modelo.

\section{CONSIDERAÇÕES FINAIS}

O presente artigo buscou fornecer subsídios teóricos que contribuam para uma melhor compreensão do modelo de dados BIBFRAME, constatou-se que em nível conceitual o BIBFRAME possui 3 Entidades principais que podem ser descritas sucintamente como: Work (Obra) representa o nível mais alto de abstração e reflete a essência conceitual do recurso catalogado; Instance (Instância) caracteriza uma manifestação e expressão de uma Obra; Item (Item) corporifica uma instância, de forma física ou virtual. Embora a entidade Instância possua algumas particularidades, tais Entidades foram definidas a partir do Grupo 1 dos FRBR. 
Para a representação formal as Entidades BIBFRAME são associadas a Classes RDF. Além das 3 Classes correspondentes as Entidades principais o vocabulário BIBFRAME provê dezenas de outras Classes e Subclasses para categorizar diferentes tipologias de recursos, possibilitando representar, por exemplo, Registros de Autoridade; Conceitos; Lugares; e praticamente qualquer Entidade do mundo real que possa ser associada a um identificador controlado e possua um conjunto específico de atributos. Para a descrição formal dos atributos e relacionamentos dos recursos são utilizadas Propriedades que se caracterizam como metadados dos recursos.

Com base na análise realizada constatou-se que que modelo BIBIFRAME oferece um impulso para o reposicionamento das bibliotecas no cenário contemporâneo, contribuindo para o fortalecimento de atividades relacionadas a identificação e formalização dos relacionamentos entre recursos disponíveis na Web. A partir do uso de identificadores associados a links 0 BIBFRAME permite um número ilimitado de possibilidades de acesso aos recursos bibliográficos, favorecendo maior visibilidade do acervo, e contribui para a validação e enriquecimento semânticos dos dados.

À medida que a Web evolui para uma "Web de Dados Interligados" é possível comprovar a importância da representação formal dos relacionamentos entre os recursos. Bibliotecários sempre foram especialistas em identificar e descrever relações, porém tradicionalmente sua atuação estava limitada aos acervos das bibliotecas. O modelo BIBIFRAME possibilita compartilhar este conhecimento especializado além dos limites das bibliotecas por meio da formalização dos relacionamentos entre recursos disponíveis na Web, contribuindo para uma mudança de paradigma em relação à forma como encaramos os processos de controle, troca e compartilhamento de recursos informacionais. 


\section{REFERÊNCIAS}

ASSUMPÇÃO, F.S.; SANTOS, P.L.V.A.C. A importância do controle de autoridade: uma abordagem baseada nos objetivos e nas funções dos catálogos. In: ENCONTRO NACIONAL DE CATALOGADORES, 1., 2012, Rio de Janeiro; ENCONTRO DE ESTUDOS E PESQUISAS EM CATALOGAÇÃO, 3., 2012, Rio de Janeiro. Disponível em: <http://eprints.rclis.org/18843>. Acesso em: 06 out. 2015.

BURGER, Robert. Authority work: the creation, use, maintenance, and evaluation of authority records and files. Littleton: Libraries Unlimited, 1985.

ECO, H. Muito além da Internet. 2003. Disponível em $<$ http://www.ofaj.com.br/textos_conteudo.php?cod=16>. Acesso em: $16 \mathrm{dez}$. 2015.

GORMAN, M. Authority Control in the Context of Bibliographic Control in the Electronic Environment. In: International Conference Authority Control: Definition and International Experiences, Florença, 2003. Disponível em: $<$ http://eprints.rclis.org/4164>. Acesso em: 06 out. 2015.

HALLO, M.; LUJA'N-MORA, S.; MATÉ, A.; TRUJILLO, J. Current state of Linked Data in digital libraries. Journal of Information Science, 2016, Vol. 42 n.2 p.117-127.

INTERNATIONAL FEDERATION OF LIBRARY ASSOCIATIONS AND INSTITUTIONS. Guidelines for Online Public Access Catalogue (OPAC) displays: final report: may 2005. München: K. G. Saur, 2005.

INTERNATIONAL FEDERATION OF LIBRARY ASSOCIATIONS - IFLA. Statement of international cataloguing principles. 2009. Disponível em: $<$ http://www.ifla.org/files/cataloguing/icp/icp_2009-en.pdf>. Acesso em: 16 jun. 2014.

LIBRARY OF CONGRESS. Bibliographic Framework as a Web of Data: Linked data model and supporting services. Washington, 2012. Disponível em: <http://www.loc.gov/bibframe/pdf/marcld-report-11-21-2012.pdf >. Acesso em: 9 mai. 2016.

MITCHELL, E. T. Building Blocks of Linked Open Data in Libraries. Library Technology Reports: Library Linked Data: Research and adoption 49, no. 5. July. 2013.

MODESTO, J.F. O acervo da biblioteca está redondo, deixe-o em forma com o marc. OFAJ, Londrina, Abril. 2007. Disponível em: <http://www.ofaj.com.br/colunas_conteudo.php?cod=294> Acesso em: 9 mai. 2015. 
RAMALHO, R.A.S. Análise do Modelo de Dados SKOS: Sistema de Organização do Conhecimento Simples para a Web. Informação \& Tecnologia (Itec), v. 2, p. 66-79, 2015. Disponível em: <http://periodicos.ufpb.br/ojs/index.php/itec/article/view/25995> Acesso em: 9 mai. 2016.

\title{
Title
}

Bibframe: linked data model for libraries

\begin{abstract}
:
Introduction: The technological development of the last decade has provided new tools for modeling, representation and organization of information resources in the most different fields of knowledge. In the field of Information Science highlights the Bibliographic Framework (BIBFRAME) as a new model of representation that enables the formal description of the relationships between resources. This data model is related to research trends involving Linked Data; Data Science; Enhanced Publication and Semantic Web.

Objective: Provide theoretical subsidies that contribute for a better understanding of BIBFRAME data model, describing its entirety, and to present a discussion on the main challenges and prospects for use of this new data model in libraries.

Methodology: The methodology is characterized as exploratory and descriptive research, with a theoretical approach.

Results: They were presented the main technologies, conceptual elements and features of BIBFRAME model and reported the challenges and prospects of using this new data model in libraries.

Conclusion: The BIBFRAME model contributes to a paradigm shift in relation to how do we envision the processes of control, exchange and sharing in informational resources, providing a boost to the repositioning of libraries in the contemporary scenery in order to strengthen activities related to identification and formalization of relationships between resources available in web.
\end{abstract}

Key Words: Knowledge Organization Systems. Information and Communication Technologies. Data Modeling. Ontologies. Semantic Web.

\section{Titulo}

Bibframe: modelo de datos enlazados para bibliotecas

Resumen:

Introducción: El desarrollo tecnológico de la última década ha proporcionado nuevas herramientas para el modelado, representación y organización de los recursos de información en diferentes áreas del conocimiento. En el campo de ciencias de la información se destaca el Bibliographic Framework (BIBFRAME) como un nuevo modelo de datos que permite la descripción formal de las relaciones entre recursos. Este modelo está relacionado con las líneas de investigación que involucran Datos Enlazados; Ciencia de Datos; Publicación Extended y Web Semántica. extendidos.

Inf. Inf., Londrina, v. 21, n. 2, p. 292 - 306, maio/ago., 2016. 
Objetivo: Proveer subsidios teóricos que contribuyen a una mejor comprensión del modelo de datos BIBFRAME, describiendo sus elementos y presenta una discusión sobre los principales desafíos y perspectivas de uso de desde nuevo modelo de datos en las bibliotecas.

Metodología: investigación exploratoria y descriptiva, con enfoque teórico.

Resultados: Se presentaron las tecnologías clave, elementos conceptuales y características del modelo BIBFRAME e reportado los retos y perspectivas de uso de este nuevo modelo de datos en las bibliotecas.

Conclusiones: El modelo BIBFRAME contribuye a un cambio de paradigma en relación con la forma en que vemos los procesos de controle, intercambio y compartir de los recursos informacionales, proporcionando un impulso al reposicionamiento de las bibliotecas en el escenario contemporáneo con la finalidad de fortalecer actividades relacionados con la identificación y formalización de las relaciones entre los recursos disponibles en la web.

Palabras clave: Sistemas de Organización del Conocimiento. Tecnologías de Información y Comunicación. Modelado de datos. Ontologías. Web Semántica.

Enviado em: 17.07.2016.

Aceito em: 20.11.2016. 